\title{
El dominio afectivo en el aprendizaje de las Matemáticas.
}

\section{Nuria Gil Ignacio, Eloísa Guerrero Barona y \\ Lorenzo Blanco Nieto.}

\section{España}

eloisa@unex.es 


\section{Resumen}

Introducción. La finalidad de este trabajo es analizar las creencias, las actitudes y las reacciones emocionales que los estudiantes experimentan en el proceso de aprendizaje de las matemáticas. El objetivo es conocer si las creencias, actitudes y atribuciones positivas acerca de sí mismos como aprendices son una fuente de motivación y expectativas de éxito ante la materia.

Método. Para ello, hemos empleado una muestra de 346 alumnos de segundo ciclo de Educación Secundaria Obligatoria (ESO) de Institutos de Badajoz, a los que se les aplicó un cuestionario sobre creencias y actitudes acerca de las matemáticas.

Resultados y Discusión. Concluimos que el género no guarda relación con el autoconcepto matemático.

Palabras clave: creencias, actitudes, emociones, autoconcepto matemático, secundaria, género y aprendizaje de las matemáticas. 


\section{Introducción}

Un problema persistente en la comprensión del afecto en la enseñanza y aprendizaje de las Matemáticas ha sido encontrar una definición clara de qué es el afecto o el dominio afectivo (Gómez-Chacón, 2000). Durante una larga época, los estudios sobre la dimensión afectiva en matemáticas estuvieron limitados al estudio de las actitudes. Sin embargo, en estas dos últimas décadas se ha ampliado al estudio de las creencias y reacciones emocionales (McLeod, 1994). Este nuevo enfoque de la dimensión afectiva, auspiciado en gran medida por los trabajos de McLeod (1988, 1992, 1994), pone de manifiesto que las cuestiones afectivas juegan un papel esencial en el proceso de enseñanza-aprendizaje de las matemáticas, y que algunas de ellas están fuertemente arraigadas en el sujeto y no son fácilmente desplazables por la instrucción.

Aprender matemáticas se ha convertido en una necesidad para desenvolverse adecuadamente en la compleja sociedad actual, donde los avances tecnológicos y la creciente importancia de los medios de comunicación hacen necesaria la adaptación de las personas a las nuevas situaciones derivadas del cambio social. Es un hecho que, a pesar de su utilidad e importancia, las matemáticas suelen ser percibidas y valoradas por la mayor parte de los alumnos como una materia difícil, aburrida, poco práctica, abstracta, etc., cuyo aprendizaje requiere una "capacidad especial", no siempre al alcance de todos. Estamos firmemente convencidos de que estas creencias influyen en el hecho de que un porcentaje considerado de los suspensos en la Educación Secundaria Obligatoria (E.S.O.) corresponda al área de matemáticas, materia en la que se concentra un elevado porcentaje de dificultades y fracasos académicos, convirtiéndose en un importante filtro selectivo del sistema educativo.

Desde nuestro punto de vista, son muchos los alumnos que generan en el transcurso de su vida académica actitudes negativas hacia las matemáticas, manifestando, en ocasiones, una auténtica aversión y/o rechazo hacia esta disciplina. Para una mayoría de los estudiantes, esta materia no es una fuente de satisfacción, sino de frustración, desánimo y angustia. A muchos de ellos, incluyendo a algunos de los más capacitados, les desagradan y agobian las matemáticas. 
De acuerdo con esto, ya en el Informe Cockcroft (1985) se puso de manifiesto cómo hasta qué punto la necesidad de emprender una simple y fácil tarea matemática podría provocar sentimientos de ansiedad, impotencia, miedo e incluso culpabilidad. Por ello, resulta necesario comprender y analizar cómo el estudiante, al aprender matemáticas y al interactuar con su entorno, interioriza determinadas creencias y valoraciones negativas o positivas hacia ellas y hacia él mismo como aprendiz, lo cual le va a generar éxitos o fracasos ante la consecución de los logros matemáticos. Ante esta situación, muchos estudiantes, pensando que "no están hechos para las matemáticas" acaban rechazándolas, pues las consideran un "lastre" del que tienen que librarse en cuanto puedan mediante la opcionalidad en la ESO y en el Bachillerato.

En este sentido, entendemos que los altos índices de fracaso escolar en el área de matemáticas exigen el estudio de la influencia de los factores afectivos y emocionales en el aprendizaje matemático, ya que pueden explicar la ansiedad que siente el alumno ante la resolución de problemas, su sensación de malestar, de frustración, de inseguridad, el bajo autoconcepto que experimenta, etc., que, frecuentemente, le impiden afrontar con éxito y eficacia las tareas matemáticas.

Según Blanco y Guerrero (2002) la historia repetida de fracasos lleva a los alumnos a dudar de su capacidad intelectual en relación con las tareas matemáticas y llegan a considerar sus esfuerzos inútiles, manifestando sentimientos de indefensión o pasividad. Por ello, se sienten frustrados y abandonan rápidamente ante la dificultad. Esta situación determina nuevos fracasos que refuerza la creencia de que efectivamente son incapaces de lograr el éxito, desarrollándose una actitud negativa que bloquea sus posteriores oportunidades de aprendizaje.

Centrándonos en el dominio afectivo en Matemáticas, el pionero en este ámbito ha sido McLeod (1989b), refiriéndose al mismo como un extenso rango de sentimientos y humores (estados de ánimo) que son generalmente considerados como algo diferente de la pura cognición e incluye como componentes específicos de este dominio las actitudes, creencias y emociones. Atendiendo a las creencias, en la literatura reciente sobre el aprendizaje de las matemáticas, las investigaciones sobre la influencia de las creencias ocupan un lugar destacado. McLeod (1992) diferencia cuatro ejes con relación a las creencias: sobre las matemáticas (el objeto), sobre uno mismo, sobre la enseñanza 
de la matemática y sobre el contexto en el que acontece la educación matemática (contexto social). Dicho autor señala dos categorías de las creencias que principalmente parecen tener influencia en los aprendices de matemáticas: creencias acerca de las matemáticas, que generalmente involucran poco componente afectivo, pero constituyen una parte importante del contexto en el que se desarrolla el afecto, y creencias del estudiante (y del profesor), acerca de sí mismo y su relación con la matemática, que poseen un fuerte componente afectivo, incluyendo las creencias relativas a la confianza, el autoconcepto y la atribución causal del éxito y fracaso escolar (McLeod, 1989b).

Las creencias relativas al autoconcepto del estudiante como aprendiz de matemáticas es, según Gómez-Chacón (2000), una de las variables que más influye en el aprendizaje y en la enseñanza de las matemáticas y está relacionada con sus actitudes, su perspectiva del mundo matemático y con su identidad social. Los elementos más importantes de este constructo son los conocimientos subjetivos y las emociones referidas a los siguientes aspectos: el interés en matemáticas, la eficiencia en matemáticas, la motivación y el placer con las matemáticas, la atribución causal de éxito o fracaso escolar y el autoconcepto como miembro de un determinado grupo social.

Gómez-Chacón (1997) afirma que el autoconcepto matemático hace referencia a la autoimagen de la persona con respecto a cómo se percibe y se valora al aprender matemáticas. Además, añade que es un aspecto del aprendizaje vinculado a las creencias personales relativas al mundo de las matemáticas, esto es, a las ideas, juicios, creencias $\mathrm{y}$ atribuciones de la persona que ha ido conformando durante su proceso de escolarización en el entorno de aprendizaje. McLeod (1992), el autoconcepto del alumno como aprendiz de matemáticas debe concebirse como una subestructura derivada de la estructura de creencias, y a la vez es uno de los descriptores básicos del dominio afectivo en matemáticas, que guarda una estrecha relación con las emociones, las actitudes, las motivaciones, las expectativas personales y las atribuciones.

Con frecuencia, los estudiantes con dificultades de aprendizaje en matemáticas, debido a su reiterada experiencia de fracaso son los que muestran patrones atribucionales más desadaptativos. Al dudar de sus capacidades, exageran la magnitud de sus deficiencias tendiendo a atribuir sus fracasos a su falta de capacidad. Asimismo, muestran bajas expectativas de éxito y abandonan fácilmente frente a las dificultades. Cuando tienen éxito lo atribuyen a la facilidad del problema, a la ayuda del profesor, a 
la de los compañeros o a la suerte, percibiendo los fracasos continuados como confirmación de su baja capacidad. Las creencias negativas respecto a sí mismos como aprendices les impiden mejorar su rendimiento en matemáticas, pues creen que el rendir bien está por encima de sus posibilidades (Chapman, 1988). Según Weiner (1992), el tipo de atribuciones que realiza el alumno tendrá repercusiones tanto a nivel cognitivo (expectativas) como a nivel afectivo-emocional (autoconcepto), lo que determinará su motivación y su grado de implicación en la realización de las actividades matemáticas. En cuanto a las actitudes de los estudiantes hacia el aprendizaje matemático, van a estar determinadas por las características personales del estudiante, relacionadas con su autoimagen académica y la motivación de logro, condicionando su posicionamiento hacia determinadas materias curriculares y no otras.

En la concepción de McLeod, las actitudes abarcan una perspectiva multidimensional de diferentes clases de matemáticas y rango de sentimientos acerca de cada una de ellas. Guerrero, Blanco y Vicente (2002) opinan que rechazo, negación, frustración, pesimismo y evitación son algunas de las manifestaciones actitudinales y comportamentales que muchos alumnos manifiestan cuando afrontan la actividad académica, bien genéricamente (sobre todo en alumnos con fracaso escolar crónico) bien ante tareas específicas como las matemáticas. El trabajo de Callahan (1971) constituye un referente que marca el inicio del crecimiento de la preocupación sobre la relación de las actitudes de los alumnos con respecto a las matemáticas.

Otras investigaciones sobre actitudes hacia las matemáticas han sido las llevados a cabo por autores como Fennema y Sherman (1976, 1978), Whitley (1979), Wolleat, Ponte, Becker y Fennema (1980), Hannafin (1981), Schofield (1982), Haladyna, Shaughnessy y Shaughnessy (1983), Minato (1983), Smith (1985), Gairín (1990), Camacho, Hernández y Socas (1995), Carbonero, Martín y Arranz (1998), Hernández y Socas (1999), Hernández, Palarea y Socas (2001) y Cubillo y Ortega (2002). Sin embargo, son escasos los estudios sobre dimensión afectiva y el aprendizaje de la matemática, y son más raros aún los relativos al estudio de las emociones. Las emociones aplicadas al ámbito matemático han sido analizadas primeramente por Mandler (1989a), y por autores como McLeod y Adams (1989), Goldin (1988a) y Debellis y Goldin (1991, 1993). 
De acuerdo con Gómez Chacón (2000), las reacciones emocionales son el resultado de discrepancias entre lo que el sujeto espera y lo que experimenta en el momento en que se produce la reacción. Al aprender matemáticas el estudiante recibe continuos estímulos asociados a las matemáticas. Ante ellos reacciona emocionalmente de forma positiva o negativa, reacción que está condicionada por sus creencias acerca de sí mismo y acerca de las matemáticas. Si ante situaciones similares, repetidamente, le produce la misma clase de reacciones afectivas, la activación de la reacción emocional (satisfacción, frustración,...) puede ser automatizada y se solidifica en actitudes. De este modo, los afectos ejercen una influencia decisiva en el aprendizaje y en cómo los alumnos perciben y consideran las matemáticas, así como en la propia visión de sí mismos como aprendices, a la vez que constituyen un elemento clave que influye en su conducta (Gil, 2003).

Al ser la dimensión afectiva en Educación Matemática un campo de estudio muy amplio, en este artículo nos vamos a centrar exclusivamente en las creencias de los alumnos acerca de sí mismos como aprendices de matemáticas, por su influencia en el rendimiento académico, en la motivación, en las atribuciones causales y en las expectativas del estudiantado.

Así, la finalidad que ha impulsado este estudio es reconocer la importancia que los factores afectivos juegan en el éxito y/o fracaso del aprendizaje matemático, con la intención de promover actitudes y creencias positivas en el alumnado que redunden en la mejora del rendimiento y de las expectativas de logro hacia esta materia. En este sentido, nos hemos propuesto como objetivo describir las creencias que los alumnos tienen sobre sí mismos como aprendices de matemáticas. Partimos de la hipótesis de existe una estrecha relación entre el género del alumnado y las creencias acerca de sí mismos como aprendices de matemáticas.

\section{Método}

\section{Participantes}

La muestra de estudio se ha obtenido mediante un muestreo probabilístico en dos etapas. En primer lugar, se han seleccionado aleatoriamente una serie de institutos de cada uno de los estratos (carácter público o privado-concertado y zona en que se 
ubican; zona centro o barriadas), y se han considerado como unidad de estudio los grupos de alumnos de cada centro, de manera que se garantice la representatividad de las zonas. En la segunda etapa, para cada uno de los institutos de la muestra se han seleccionado aleatoriamente grupos de alumnos de los cursos de $3^{\circ}$ y $4^{\circ}$ de ESO.

La muestra se ha seleccionado por afijación proporcional y su tamaño se ha determinado utilizando fórmulas de muestreo, teniendo en cuenta el tamaño de la población. Todo ello a un nivel de confianza del 95\%, expresado en un coeficiente de confianza redondeado y un margen de error del 0,5\%, estimándose así que el tamaño de la muestra debía de ser aproximadamente de 300 alumnos lo que representaría un 7\% de la población.

Finalmente, la muestra quedó formada por 346 alumnos, 166 varones y 180 mujeres, con edades comprendidas entre los 13 y los 18 años, procedentes de tres Institutos públicos de Educación Secundaria de Badajoz (el 46\% de los estudiantes) y de tres privados-concertados (el 54\%), siendo 192 alumnos los que cursan de $3^{\circ}$ de ESO y 154 los que estudian $4^{\circ}$. Pertenecen a institutos situados en la zona centro de Badajoz 240 alumnos (un 69,4\%) y a centros situados en barriadas, 106 alumnos (el 30,6\%). Pertenecen a una clase sociocultural media-baja, con niveles intelectuales normales y sin handicaps físicos, psíquicos o sensoriales.

\section{Instrumentos}

El instrumento de recogida de datos empleado consistió en un cuestionario elaborado "ad hoc" sobre las creencias y actitudes del alumnado acerca de las matemáticas (Apéndice). Está compuesto por 52 ítems (con cuatro alternativas de respuesta: "de acuerdo"; "muy de acuerdo"; "en desacuerdo" y "muy en desacuerdo") agrupados en cinco bloques temáticos. Para su elaboración hemos tenido en cuenta trabajos anteriores tales como los de Callejo (1994), Camacho, Hernández y Socas (1995) y Gómez-Chacón (2000), y se han añadido otros ítems relativos a las creencias acerca del papel del profesorado de matemáticas y las que suscita el contexto sociocultural, elaborados a partir de la revisión de las fuentes bibliográficas y de los instrumentos de los autores antes mencionados. 
Por el amplio número de variables objeto de estudio, en el presente artículo sólo nos centramos en el bloque referido a las "Creencias acerca de uno mismo como aprendiz de matemáticas" (ítems 12 a 22), que hacen referencia al autoconcepto matemático, como uno de los componentes del dominio afectivo en el aprendizaje de las matemáticas. En la Tabla 1 presentamos los ítems correspondientes a este bloque y las puntuaciones medias obtenidas en cada uno de ellos.

Tabla 1: Puntuaciones obtenidas en los ítems del bloque referido a las "creencias acerca de uno mismo como aprendiz de matemáticas "(ítems 12 a 22).

12.El gusto por las matemáticas influye a la hora de escoger una determinada modalidad de bachillerato en la que estén o no presentes (puntuación media =3,15)

13.El ser buen alumno en matemáticas (sacar buenas notas, tener buena actitud) te hace sentirse más valorado y admirado por los compañeros (puntuación media $=2,23$ )

14. Si no comprendo las matemáticas, difícilmente podré asimilar y dominar otras asignaturas relacionadas con ella (como física, química, etc.) (puntuación media = 3,04)

15.Mi rendimiento en matemáticas depende en gran medida de la actitud del profesor hacia mí (puntuación media $=2,77$ )

16.Cuando dedico más tiempo de estudio a las matemáticas obtengo mejores resultados en la resolución de problemas (puntuación media $=3,13$ )

17. Cuando resuelvo un problema suelo dudar de si el resultado es correcto (puntuación media $=2,98$ )

18.Tengo confianza en mí mismo cuando me enfrento a los problemas de matemáticas (puntuación media $=2,45$ )

19. Me considero muy capaz y hábil en matemáticas (puntuación media =2,31)

20.Estoy calmado y tranquilo cuando resuelvo problemas de matemáticas (puntuación media $=2,49$ )

21. Cuando me esfuerzo en la resolución de un problema suelo dar con el resultado correcto (puntuación media $=2,73$ )

22.La suerte influye a la hora de resolver con éxito un problema de matemáticas (puntuación media $=1,91$ ) 


\section{Variables de estudio}

En lo que respecta a las variables que se han incluido en el estudio, éstas se clasifican y definen de la siguiente manera:

1) Circunstancias personales:

\section{- Género.}

2) Variables curriculares:

- Curso.

- Calificación obtenida en el área de matemáticas.

- Preferencia e importancia dada a las distintas asignaturas del currículum.

3) Autoconcepto del alumno como aprendiz de matemáticas:

- Se define por el nivel de confianza y seguridad del alumno en sus habilidades, capacidades y posibilidades para desenvolverse con éxito en la materia.

- Por las expectativas de logro relacionadas con el placer y gusto por aprender matemáticas y por seguir cursándolas en el Bachillerato, con el deseo de dominar la materia, con la valoración y reconocimiento de los demás.

- Por los procesos de atribución causal del éxito o fracaso en matemáticas (motivos que atribuyen al éxito o fracaso - profesor; dedicación; esfuerzo; suerte-).

\section{Diseño}

El presente trabajo es un estudio descriptivo exploratorio en el que se ha llevado a cabo un tipo de investigación denominado investigación por encuesta, ya que, de acuerdo con Buendía (1999), es la metodología más indicada para recoger opiniones, creencias o actitudes y es una importante alternativa cuando no es posible acceder a la observación directa por circunstancias contextuales o problemas económicos.

Dado que se trata de un tema todavía poco investigado en el ámbito español, consideramos necesario aplicar este tipo de metodología con el propósito de explorar la 
realidad inicial y poder conocer así cómo interpretan los sujetos, que forman parte de la muestra, los contenidos que aparecen en el cuestionario que se les aplica a tal efecto.

Por otro lado, el diseño metodológico empleado para establecer posibles asociaciones entre las variables de estudio consiste en un diseño correlacional, pues este tipo de diseño facilita nuestra investigación al no ser posible la intervención, la manipulación, ni la selección de niveles de la variable independiente.

\section{Procedimiento}

Una vez elaborado el instrumento de recogida de datos y realizado el estudio piloto, pasamos a una nueva fase del proceso de investigación consistente en la recogida de los datos para su posterior codificación y análisis.

En cuanto al diseño empleado en el proceso de recogida de los datos, se ha utilizado un diseño transversal, que es el más utilizado en la investigación por encuesta. En este tipo de diseño los datos se recogen, sobre uno o más grupos de sujetos de Educación Secundaria, en un solo momento temporal. Se trata del estudio en un determinado corte puntual en el tiempo, en el que se obtienen las medidas a tratar. En un primer momento, se procedió a contactar telefónicamente con los profesores de matemáticas responsables de los grupos de $3^{\circ}$ y $4^{\circ}$ de ESO de cada uno de los I.E.S. que han formado parte de la muestra, fijándose así unos días claves en los que se han llevado a cabo entrevistas con dichos profesores, con el fin de informarles acerca del propósito de nuestra investigación y obtener su consentimiento y colaboración para poder llevar acabo el estudio.

En las sucesivas reuniones con el profesorado de matemáticas se les explicó la finalidad de la investigación, se les instruyó en la aplicación del cuestionario y se les solicitó su total implicación en el proceso de recogida de datos. Los cuestionarios se han aplicado de forma colectiva en cada uno de los centros dentro del horario normal de las clases, en una atmósfera relajada y de total colaboración del alumnado y del profesorado, empleándose para su cumplimentación un tiempo de entre 15 y 20 minutos. La secuencia de instrucción a cargo del profesorado ha consistido en todos los casos en la presentación del cuestionario a los alumnos, informándoseles acerca de los 
fines y motivos de la investigación, de las correspondientes instrucciones para su correcta cumplimentación y del tiempo estimado para ello.

En cuanto al tratamiento estadístico de datos, tras ser sometidos a los necesarios procesos de depuración, codificación y grabación en soporte informático, se han analizado utilizando el paquete estadístico SPSS 10.0. Para el análisis de los datos, efectuado a un nivel de confianza del $95 \%$ y con un margen de error del $5 \%$, se han utilizado los siguientes estadísticos descriptivos: frecuencias, porcentajes, medias aritméticas y desviaciones típicas. Asimismo, para el análisis de la correlación entre pares de variables se ha empleado el coeficiente de correlación de Pearson.

\section{Resultados}

Los datos obtenidos muestran que el rendimiento de las alumnas en Matemáticas es ligeramente inferior al de los alumnos. De este modo, atendiendo al rendimiento del alumnado en matemáticas en función de si tienen pendiente la asignatura o no, el 23,9\% de las alumnas sí tiene pendiente la asignatura, frente a un $20,5 \%$ de los varones. Además, con respecto a las calificaciones de los alumnos en esta materia, predomina el porcentaje de alumnos que obtiene la calificación de "Sobresaliente" (17,5\%), frente a un $14,4 \%$ de las alumnas. Sumando las calificaciones Bien, Notable y Sobresaliente los varones obtienen un $60,3 \%$ frente a un $54,4 \%$ de las mujeres.

Centrándonos en el bloque relativo a las creencias acerca de uno mismo como aprendiz de matemáticas, y atendiendo al grado de confianza, tranquilidad y calma en el proceso de resolución de problemas (ítems 18 y 20, respectivamente), el 37,3\% de los alumnos manifiestan no tener confianza en sí mismos cuando se enfrentan a la resolución de problemas, frente a un $51,1 \%$ de las alumnas. También, ellos se sienten más calmados y tranquilos cuando resuelven problemas de matemáticas, pues sólo un $8,4 \%$ se muestra "muy en desacuerdo" con esta afirmación, frente al 17,2\% de las alumnas.

En cuanto al sentimiento de capacidad y habilidad en matemáticas, el porcentaje de alumnos que se siente muy capaz y hábil en la materia (un 42,2\%) es ligeramente 
superior al de las alumnas (un 30,6\%). Asimismo, cuando se trata de valorar el grado de seguridad del alumnado en el proceso de resolución de problemas (ítem 17: "cuando resuelvo un problema suelo dudar si el resultado es correcto"), también la proporción de alumnos que se siente seguro (el 18,7\%) es superior a la de las alumnas (el 13,9\%).

En cuanto al ítem 12, "el gusto por las matemáticas influye a la hora de escoger una determinada modalidad de Bachillerato en la que estén o no presentes", a las alumnas les condiciona más que a los alumnos el gusto y la atracción por las matemáticas a la hora de escoger y decantarse por una modalidad de Bachillerato. Sin embargo, la correlación efectuada pone de manifiesto que no existen diferencias significativas entre ambos géneros $(r x y=0,017 ; p=0,750)$.

En el análisis del ítem 14, "si no comprendo las matemáticas, difícilmente podré asimilar y dominar otras asignaturas relacionadas con ellas", el deseo de dominio y las expectativas de éxito en la materia quedan patentes. De este modo, el 39,3\% del estudiantado considera que si no comprenden ni dominan las matemáticas no podrán asimilar y comprender otras materias relacionadas con ellas, mientras que un $7,2 \%$ no está de acuerdo, no apreciándose diferencias significativas en cuanto al género ( $r x y=$ $0,001 ; p=0,981)$.

Por su parte, el 27,1\% de los alumnos y el 23,3\% de las alumnas opinan que el ser buen/a alumno/a en matemáticas (sacar buenas notas, tener buena actitud) les hace sentirse más valorados/as y admirados/as por sus compañeros (ítem 13), frente al 49,4\% y el 55,6\%, respectivamente, que expresan estar "muy en desacuerdo" con este ítem. Este resultado viene a poner de manifiesto que, a diferencia de las chicas, para los chicos el ser bueno en la materia reporta una mayor valoración social por parte de sus compañeros.

Finalmente, en relación a las atribuciones causales del éxito y/o fracaso en matemáticas, el 39,2\% de los alumnos consideran que la actitud del profesor no condiciona su éxito o fracaso en matemáticas, mientras que el 41,7\% de las alumnas sí los atribuye al comportamiento del profesor (ítem 15: "mi rendimiento en matemáticas depende en gran medida de la actitud del profesor hacia mí”). Asimismo, tan sólo el $12 \%$ de los chicos, frente al $20 \%$ de las chicas, opina que el factor suerte influye en su 
rendimiento en la materia (ítem 22 "la suerte influye a la hora de resolver con éxito un problema de matemáticas").

Sin embargo, sí se observan diferencias estadísticamente significativas en cuanto al género en la atribución causal del éxito a la dedicación ( $r x y=0,119 ; p=0,026)$ (ítem 16, "cuando dedico más tiempo de estudio a las matemáticas obtengo mejores resultados en la resolución de problemas") y al esfuerzo ( $r x y=-0,143 ; p=0,008)$ (ítem 21 "cuando me esfuerzo en la resolución de un problema suelo dar con el resultado correcto").

\section{Discusión}

Si bien nuestro objetivo inicial trataba de poner de manifiesto la relevancia de los factores afectivos en el aprendizaje de las matemáticas, los resultados del análisis sobre las creencias acerca de uno mismo como aprendiz de matemáticas revelan que los alumnos de la muestra tienen un autoconcepto matemático más ajustado que las alumnas. Partiendo de la base teórica de que el autoconcepto y el rendimiento se influyen y se determinan mutuamente, cabe destacar que el rendimiento de las alumnas en matemáticas es inferior al de los alumnos. Los datos mostrados asumirían esta relación, ya que el rendimiento de las alumnas es inferior, así como su autoconcepto. En este sentido, para tener un buen rendimiento, es necesario, aunque no suficiente, que el alumno tenga un concepto positivo de su valía y de su competencia para el trabajo escolar, y que, al mismo tiempo, el éxito en las actividades académicas le informe de que es competente y capaz, lo que contribuirá a consolidar un autoconcepto positivo.

Predomina el porcentaje de alumnos varones que obtiene la calificación de "Sobresaliente", siendo la proporción de alumnas superior a la de los varones. De este modo, parece ser que el obtener buenas calificaciones en la materia motiva a los estudiantes y mejora su autoconcepto como aprendices de la misma, dado que se sienten más competentes y capaces, y, a la vez, ese sentimiento de seguridad, valía y simpatía en y por la materia, refuerza su autoconcepto como aprendices y favorece el rendimiento académico. 
En relación con los datos anteriores, Sampascual, Navas y Castejón (1994), afirmaron que los alumnos que cuentan con un rendimiento alto o satisfactorio, a diferencia de los que tienen un rendimiento bajo o insatisfactorio, gozan de un autoconcepto más positivo, de unas expectativas más elevadas, obtienen mejores calificaciones y realizan más atribuciones al esfuerzo. Por una parte, los alumnos manifiestan tener más confianza en sí mismos, sentirse más seguros, más calmados y tranquilos, más capaces y hábiles en esta materia que las alumnas. De este modo, dichas actitudes y reacciones emocionales ante las matemáticas influyen positivamente en su percepción hacia la disciplina, así como en su autoconcepto matemático y en sus expectativas de logro. En este sentido, autores como Lester, Garofalo y Kroll (1989) señalan que las creencias de los alumnos acerca de la resolución de los problemas tienden a afectar a su autoconfianza en esa actividad, aumentándola o disminuyéndola.

Por otra parte, a las alumnas les influye más el gusto por las matemáticas que a los alumnos a la hora de escoger una determinada modalidad de Bachillerato. Este hecho puede deberse a varias razones: ellas sienten más rechazo por esta materia, experimentan más ansiedad, obtienen calificaciones más bajas que los chicos, tienen menores expectativas de éxito, etc. Del mismo modo, para los alumnos el ser bueno en matemáticas (sacar buenas notas, tener buena actitud, etc.) reporta una mayor valoración social por parte del resto de compañeros y su deseo de dominio y las expectativas de éxito en la materia quedan patentes, pues consideran que si no comprenden ni dominan las matemáticas no podrán asimilar y comprender otras materias relacionadas con ellas.

Los estudios llevados a cabo por Fennema y Sherman $(1976$, 1978) muestran diferencias significativas entre alumnos y alumnas, presentando éstos un aprovechamiento superior al de las chicas, ligado a una mayor autoconfianza de su parte en el trabajo en matemáticas. En caso de igual aprovechamiento, las chicas presentaban menor autoconfianza que los chicos. En cambio, los resultados obtenidos en nuestro trabajo ponen de manifiesto que los chicos consiguen un rendimiento más alto en esta disciplina y tienen más confianza y seguridad en sí mismos a la hora de enfrentarse a las tareas matemáticas que las chicas.

Con respecto a la atribución causal del éxito y/o fracaso en matemáticas, a diferencia de las alumnas, los alumnos encuestados creen que ni la actitud del profesor, 
ni la suerte condicionan el rendimiento en esta materia. En cambio, sí consideran que la dedicación y el esfuerzo son factores esenciales a la hora de alcanzar el éxito en la disciplina. Estas atribuciones al esfuerzo y la dedicación hacen que los estudiantes se enfrenten a las tareas matemáticas con un autoconcepto positivo, ya que si piensan que tienen éxito porque se esfuerzan o que fracasan porque no le dedican mucho tiempo, éste no se verá mermado.

Este resultado tiene cierta conexión con los obtenidos por Pintrich, Anderman y Klobucar (1994) y por Navas, Sampascual y Castejón (1994), donde se pone de relieve la relación entre la atribución del éxito a causas internas y controlables (esfuerzo, capacidad, dedicación) y los aspectos motivacionales y cognitivos. Los resultados de estos estudios han confirmado que aquellos estudiantes que atribuyeron el éxito a causas internas y al control se mostraron menos ansiosos, con más expectativas de éxito, más orientados al dominio, autoeficaces y metacognitivos y, además, rindieron mejor. Adicionalmente, los estudiantes que creían que su fracaso era debido a factores inestables e incontrolables estaban menos orientados al dominio, eran menos eficaces, tenían menores expectativas de logro y más bajo rendimiento académico.

Por el contrario, nuestros datos difieren de los obtenidos por Wolleat et al. (1980), quienes concluyeron que las chicas explicaban su éxito por su esfuerzo, mientras que ellos lo justificaban por sus capacidades, y al explicar el fracaso las chicas atribuían más que los chicos esa cuestión a la dificultad de la tarea. Nuestros resultados indican que los chicos atribuyen sus éxitos y/o fracasos en matemáticas a su esfuerzo y sentimiento de competencia, mientras que las chicas a la suerte y a la actitud del profesor.

\section{Conclusiones}

A la vista de los resultados obtenidos, podemos extraer como conclusión que las creencias acerca de sí mismo como aprendices de matemáticas (confianza y seguridad en sí mismos, atribuciones causales, expectativas de logro, etc.) no se relacionan con género del alumnado. Por esta razón, rechazamos la hipótesis relativa a la existencia de una relación directa y positiva entre el género y el autoconcepto matemático. 
Para concluir, presentamos una serie de propuestas, que creemos necesario tener en cuenta de cara a futuros estudios relacionados con el tema. En primer lugar, sería de gran interés elaborar "programas de alfabetización emocional en educación matemática", con el fin de promover el cambio de actitudes, creencias y emociones en E.S.O. hacia las matemáticas y su aprendizaje. Además, una mejora de las actitudes hacia las matemáticas ha de pasar necesariamente por un cambio de la imagen que se tiene de esta materia, a la que consideramos no es ajena la metodología didáctica que se utiliza, así como por una mejora de las relaciones entre profesores y alumnos.

En segundo lugar, creemos que es una necesidad fomentar la colaboración entre los orientadores y los profesores de matemáticas en el campo del dominio afectivo, debido a su influencia en la calidad del aprendizaje escolar, a través de la puesta en marcha de proyectos y programas de prevención e intervención en dificultades de aprendizaje en matemáticas y de educación emocional en esta área del conocimiento, que favorezcan la atracción y gusto por la disciplina y mejoren las actitudes, creencias y reacciones emocionales que experimentan los estudiantes ante su aprendizaje.

En tercer lugar, en estudios posteriores sería importante profundizar en la relación entre variables como la zona en que se ubica el centro al que asisten los alumnos, el tipo de centro, etc., y las creencias acerca de la naturaleza y aprendizaje de las matemáticas, acerca del papel del profesorado en el proceso de enseñanzaaprendizaje y las creencias suscitadas por el contexto sociofamiliar (familia, grupo de iguales, medios de comunicación, etc.). Incluso, se podría extender este estudio al último ciclo de Primaria ( $5^{\circ}$ y $6^{\circ}$ curso) y comparar la influencia de los factores afectivos en el aprendizaje matemático en ambas etapas educativas. 


\section{Referencias}

Blanco, L.J. y Guerrero, E. (2002). Profesionales de las Matemáticas y Psicopedagogos. Un encuentro necesario. En $\mathrm{M}^{\mathrm{a}}$ C. Penalva, G. Torregosa y J. Valls (Coords.), Aportaciones de la Didáctica de la matemática a diferentes perfiles profesionales (pp. 121-140). Actas del V Simposio de Didáctica de las matemáticas. Universidad de Alicante.

Buendía, L. (1999). La investigación por encuesta. En L. Buendía, P. Colás y F. Hernández, Métodos de investigación en psicopedagogía (pp. 119-155). Madrid: McGraw-Hill.

Callahan, J. (1971). Adolescent Attitudes Toward Mathematics. Mathematics Teacher, $9(1), 751-755$.

Callejo, M.L.(1994). Un club matemático para la diversidad. Madrid: Narcea.

Camacho, M., Hernández, J. y Socas, M.M. (1995). Concepciones y actitudes de futuros profesores de secundaria hacia la Matemática y su enseñanza: un estudio descriptivo. En L.J. Blanco y V. Mellado. La formación del profesorado de ciencias y matemáticas en España y Portugal (pp. 81-97). Servicio de publicaciones Diputación Provincial de Badajoz.

Carbonero, M.A., Martín, L.J. y Arranz, E. (1998). Expectativas ante las matemáticas de alumnos de primer ciclo de Educación Secundaria. Revista de Psicodidáctica, $6,17-26$.

Chapman, J.W. (1988). Learning Disabled Children's Self-Concept. Review of Educational Research, 58(3), 347-371.

Cockcroft, Informe (1985). Las matemáticas sí cuentan. Madrid: MEC.

Cubillo, C. y Ortega, T. (2002). Influencia de un modelo didáctico en la opinión/actitud de los alumnos hacia las matemáticas. UNO Revista de Didáctica de las matemáticas, 31, 57-72.

Debellis, V.A. y Goldin, G.A. (1991). Interactions between cognition and affect in high school students' individual problem solving. En R.G. Underhill (Ed.), Proceedings of the Thirteenth Annual Meeting on the Psychologv of Mathemtics Education, North Americam Chapter of Internatioal Group (Vol.1, pp. 29-35). Virginia Polytechnic Institute and State University.

Debellis, V.A. y Goldin, G.A. (1993). Analysis of interactions between affect and cognition in elementary school children during problem solving. En J.R. Becker 
y B.J. Pence (Eds.). Proceedings of the Fifteenth Annual Meeting on the Psychologv of Mathemtics Education, North Americam Chapter of Internatioal Group (Vol. 2, pp 56-62). Pacific Grove, CA, USA.

Fennema, E. y Sherman, J. (1976). Fennema-Sherman Mathematics Attitudes Scales : Instrument Designed to Measure Attitudes Toward mathematics, Toward the Learning of Mathematics by Females and Males. Journal for Research in Mathematics Education, 7, 324-326.

Fennema, E. y Sherman, J. (1978). Sex-Related Differences in Mathematics Achievement and Related Factor: a Further Study. Journal for Research in Mathematics Education, 9(3), 189-203.

Gairín, J. (1990). Las actitudes en educación. Un estudio sobre la educación matemática. Barcelona: Boixareu Universitaria.

Gil, N. (2003). Creencias, actitudes y emociones en el aprendizaje matemático. Memoria de investigación de Doctorado no publicada. (Puede obtenerse en N. Gil, Universidad de Extremadura., Departamento de Psicología y Sociología de la Educación (06071). Badajoz.

Goldin, G.A. (1988). Affective representation and mathematical problem solving. En M-J. Behr; C.B. Lacampagne y M.M. Wheler (Eds.), Proceedings of the Tenth Annual Meeting on the Psychology of Mathematics Education, North American Chapter of International Group (pp. 1-7). North Illinois University: DeKalb, IL.

Gómez-Chacón, I.M. (1997). Procesos de aprendizaje en matemáticas con poblaciones de fracaso escolar en contextos de exclusión social. Las influencias afectivas en el conocimiento de las matemáticas. Tesis doctoral no publicada. Universidad Complutense.

Gómez-Chacón, I.M. (2000). Matemática emocional. Los afectos en el aprendizaje matemático. Madrid: Narcea.

Guerreo, E; Blanco, L.J. y Vicente, F. (2002). El tratamiento de la ansiedad hacia las matemáticas. En J.N. García (Coord.) Aplicaciones de intervención psicopedagógica. (229-237) Madrid. Psicología Pirámide.

Lester, F., Garofalo, J. y Kroll, D. (1989). Self-Confiance, Interest, Beliefs and Metacognition: Key Influences on Problem Solving Behavior. En D.B. McLeod y V. Adams (Eds.), Affect and Mahematical Problem Solving: A New Perspective (pp. 68-75). New York: Springer-Verlag. 
Haladyna, T., Shaughnessy, J. y Shaughnessy, J.M. (1983). A Casual Analysis of Attitude Toward Mathematics. Journal for Research in Mathematics Education, vol. 14(1), 19-29. USA.

Hannafin, M. J. (1981). Effects of Teacher and Student Goal Setting and Evaluations on Mathematics Achievement and Student Attitudes. Journal of Educational Research, 74, (5), 68-79.

Hernández, J., Palarea, M. M. y Socas, M.M. (2001). Análisis de las concepciones, creencias y actitudes hacia las Matemáticas de los alumnos que comienzan la Diplomatura de Maestro. El papel de los materiales didácticos. En M. Socas, M. Camacho y A. Morales, Formación del profesorado e investigación en educación matemática II (pp. 115-124). Departamento de Análisis matemático. Universidad de la Laguna.

Hernández, J. y Socas, M. M. (1999). Las actitudes de los alumnos hacia las matemáticas. El papel de los materiales didácticos. En M. Socas, M. Camacho y A. Morales. Formación del profesorado e investigación en Educación Matemática I (pp.105-114). Departamento de Análisis matemático. Universidad de la Laguna.

Mandler, g. (1989a). Affect and learning: Causs and consequences of emotional interactions. En D.B. McLeod y V.M. Adams (Eds.), Affect and mathematical problem solving: A new perspective (pp.3-19). New York: Springer-Verlang.

Mcleod, D.B. (1988). Affective issues in mathematical problem solving: Some theoretical considerations. Journal for Research in Mathematics Education, 19, 134-141.

Mcleod, D.B. (1989b). Beliefs, attitudes, and emotions: new view of affect in mathematics education. En D.B. McLeod y V.M. Adams (Eds.), Affect and mathematical problem solving: A new perspective (pp. 245-258). New York: Springer-Verlang.

Mcleod, D.B. (1992). Research on affect in mathematics education: A reconceptualization. En Douglas A. Grouws (Ed.), Handbook of Research on mathematics Teaching and Learning (pp.575-598). New York: Macmillan.

Mcleod, D.B. (1994). Research on affect and mathematics learning in the JRME: 1970 to the present. Journal for Research in Mathematics Education, 25(6), 637-647.

Mcleod, D.B. y Adams, V.M. (Eds.) (1989). Affect and mathematical problem solving: A new perspective. New York: Springer-Verlang. 
Minato, S. (1983). Some Mathematical Attitudinal Data on Eighth Grade Students in japan Measured by a Semantic Differential. Educational Studies in Mathematics, 14(1), 19-54.

Pintrich, P.R., Anderman, E.M. y Klobucar, C H. (1994). Intraindividual differences in motivation and cognition in students with and without learning disabilities. Journal of Learning Disabilities, 27, 360-370.

Sampascual, G., Navas, L. y Castejón, J.L. (1994). Procesos atribucionales en la Educación Secundaria Obligatoria: un análisis para la reflexión. Revista de Psicología General y Aplicada, 47, 449-459.

Schofield, H. (1982). Sex, Grade Level, and the Relationship between Mathematics Attitude and Achievement in Children. The Journal Educational Research, 75, (2), 280-284.

Smith, L. R. (1985). Presentational Behaviors and Student Achievement in Mathematics. Journal of Educational Research, 78, ( 5), 292-298.

Weiner, B. (1992). Human motivation: metaphors, theories, and research. Newbury Park, California: Sage.

Whitley, T.W (1979). The effects of Individualized Iinstruction on the Attitudes of Middle School Pupils. Journal of Educational Research, 75, 188-193.

Wolleat, P., Ponte, J., Becker, A. y Fennema, E. (1980). Sex Differences in Higth School Students Causal Attributions of Performance in Mathematics. Journal for Research in Mathematics Education, 11(5), 356-366. 


\section{Apéndice}

\section{CUESTIONARIO sobre CREENCIAS y ACTITUDES acerca de las MATEMÁTICAS}

Estimado alumno/a. A continuación te presentamos un cuestionario diseñado con el objetivo de conocer tus actitudes y creencias con relación a las Matemáticas, tu experiencia de aprendizaje, así como la imagen que tienes de ti mismo como aprendiz de matemáticas. Contesta con calma y sinceramente a cada una de las preguntas y, por favor, no dejes ninguna por contestar, pues tus respuestas son de suma importancia para este estudio. Si tienes alguna sugerencia o aclaración que hacer respecto al tema indícala en el apartado de observaciones.

Gracias por tu colaboración

\section{DATOS DE IDENTIFICACIÓN}

GÉNERO:
$\square$ HOMBRE
$\square$ MUJER

EDAD:

CURSO:

CENTRO:

TIPO DE CENTRO: $\quad \square$ PÚBLICO $\quad \square$ PRIVADO

SI HAS REPETIDO ALGÚN CURSO INDICA CUÁL:

¿TIENES PENDIENTE LA ASIGNATURA DE MATEMÁTICAS? $\square$ SÍ $\square$ NO

CALIFICACIÓN OBTENIDA EN EL ÁREA DE MATEMÁTICAS (CURSO ANTERIOR):

ASIGNATURA PREFERIDA EN EL PRESENTE CURSO ACADÉMICO:

\section{NIVEL DE INSTRUCCIÓN DE LOS PADRES:}

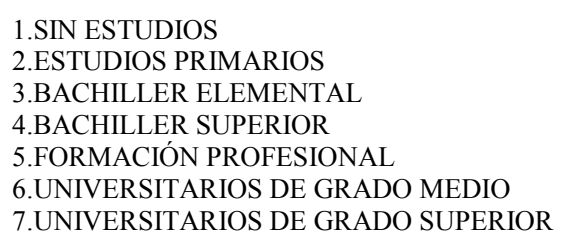

\section{PROFESIÓN/SITUACIÓN LABORAL DE LOS PADRES:}

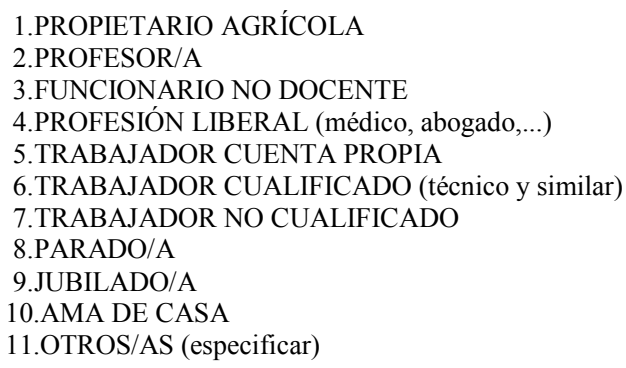

(rodea con un círculo)

\begin{tabular}{|c|c|}
\hline PADRE & MADRE \\
1 & 1 \\
2 & 2 \\
3 & 3 \\
4 & 4 \\
5 & 5 \\
6 & 6 \\
7 & 7 \\
\hline
\end{tabular}

\begin{tabular}{|c|r|}
\hline PADRE & MADRE \\
1 & 1 \\
2 & 2 \\
3 & 3 \\
4 & 4 \\
5 & 5 \\
6 & 6 \\
7 & 7 \\
8 & 8 \\
9 & 9 \\
10 & 10 \\
11 & 11 \\
\hline
\end{tabular}


NÚMERO DE HERMANOS (incluido el/a alumno/a):

\section{INSTRUCCIONES}

Para contestar el cuestionario has de marcar con una $\mathbf{X}$ la opción de respuesta que consideres más oportuna. Las escalas de valores son las siguientes:

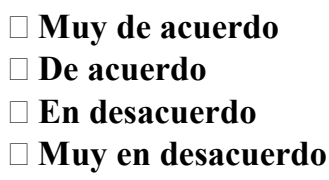

1. Las matemáticas son útiles y necesarias en todos los ámbitos de la vida $\square$ Muy de acuerdo $\square$ De acuerdo $\square$ En desacuerdo $\quad \square$ Muy en desacuerdo

2. Las matemáticas son difíciles, aburridas y alejadas de la realidad

$\square$ Muy de acuerdo $\quad \square$ De acuerdo $\quad \square$ En desacuerdo $\quad \square$ Muy en desacuerdo

3. En matemáticas es fundamental aprenderse de memoria los conceptos, fórmulas y reglas

$\square$ Muy de acuerdo $\square$ De acuerdo $\square$ En desacuerdo $\quad \square$ Muy en desacuerdo

4. Casi todos los problemas de matemáticas se resuelven normalmente en pocos minutos, si se conoce la fórmula, regla o procedimiento que ha explicado el profesor o que figura en el libro de texto

$\square$ Muy de acuerdo $\square$ De acuerdo $\square$ En desacuerdo $\quad \square$ Muy en desacuerdo

5. Las únicas matemáticas que me interesan son las que entran en el examen, porque son las más importantes y las que tengo que conocer

$\square$ Muy de acuerdo $\square$ De acuerdo $\square$ En desacuerdo $\square$ Muy en desacuerdo

6. La mejor forma de aprender matemáticas es a través del estudio individual

$\square$ Muy de acuerdo $\quad \square$ De acuerdo $\quad \square$ En desacuerdo $\quad \square$ Muy en desacuerdo

7. El resultado al que llego tras intentar resolver un problema es más importante que el proceso que he seguido

$\square$ Muy de acuerdo $\quad \square$ De acuerdo $\quad \square$ En desacuerdo $\quad \square$ Muy en desacuerdo

8. Sabiendo resolver los problemas que propone el profesor en clase, es posible solucionar otros del mismo tipo si sólo les han cambiado los datos

$\square$ Muy de acuerdo $\square$ De acuerdo $\square$ En desacuerdo $\quad \square$ Muy en desacuerdo

9. Las destrezas o habilidades que utilizo en clase para resolver problemas no tienen nada que ver con las que utilizo para resolver problemas en la vida cotidiana

$\square$ Muy de acuerdo $\square$ De acuerdo $\square$ En desacuerdo $\quad \square$ Muy en desacuerdo

10. Busco distintas maneras y métodos para resolver un problema

$\square$ Muy de acuerdo $\square$ De acuerdo $\square$ En desacuerdo $\quad \square$ Muy en desacuerdo

11. Aprendo mucho inventándome nuevos problemas

$\square$ Muy de acuerdo $\square$ De acuerdo $\square$ En desacuerdo $\square$ Muy en desacuerdo 
12. El gusto por las matemáticas influye a la hora de escoger una determinada modalidad de bachillerato en la que estén o no presentes

$\square$ Muy de acuerdo $\quad \square$ De acuerdo $\quad \square$ En desacuerdo $\quad \square$ Muy en desacuerdo

13. El ser buen/a alumno/a en matemáticas (sacar buenas notas, tener buena actitud) te hace sentirse más valorado y admirado por los compañeros

$\square$ Muy de acuerdo $\square$ De acuerdo $\square$ En desacuerdo $\quad \square$ Muy en desacuerdo

14. Si no comprendo las matemáticas, difícilmente podré asimilar y dominar otras asignaturas relacionadas con ella (como física, química, etc.)

$\square$ Muy de acuerdo $\quad \square$ De acuerdo $\quad \square$ En desacuerdo $\quad \square$ Muy en desacuerdo

15. Mi rendimiento en matemáticas depende en gran medida de la actitud del/a profesor/a hacia mí

$\square$ Muy de acuerdo $\square$ De acuerdo $\square$ En desacuerdo $\quad \square$ Muy en desacuerdo

16. Cuando dedico más tiempo de estudio a las matemáticas obtengo mejores resultados en la resolución de problemas

$\square$ Muy de acuerdo $\square$ De acuerdo $\square$ En desacuerdo $\quad \square$ Muy en desacuerdo

17. Cuando resuelvo un problema suelo dudar de si el resultado es correcto

$\square$ Muy de acuerdo $\square$ De acuerdo $\square$ En desacuerdo $\quad \square$ Muy en desacuerdo

18. Tengo confianza en mí mismo/a cuando me enfrento a los problemas de matemáticas

$\square$ Muy de acuerdo $\square$ De acuerdo $\square$ En desacuerdo $\square$ Muy en desacuerdo

19. Me considero muy capaz y hábil en matemáticas

$\square$ Muy de acuerdo $\quad \square$ De acuerdo $\square$ En desacuerdo $\quad \square$ Muy en desacuerdo

20. Estoy calmado/a y tranquilo/a cuando resuelvo problemas de matemáticas

$\square$ Muy de acuerdo $\quad \square$ De acuerdo $\square$ En desacuerdo $\quad \square$ Muy en desacuerdo

21. Cuando me esfuerzo en la resolución de un problema suelo dar con el resultado correcto

$\square$ Muy de acuerdo $\quad \square$ De acuerdo $\quad \square$ En desacuerdo $\quad \square$ Muy en desacuerdo

22. La suerte influye a la hora de resolver con éxito un problema de matemáticas

$\square$ Muy de acuerdo $\square$ De acuerdo $\square$ En desacuerdo $\quad \square$ Muy en desacuerdo

23. En clase de matemáticas los/as profesores/as emplean gran variedad de medios y ejemplos prácticos que me permiten relacionar las matemáticas con situaciones de mi vida diaria

$\square$ Muy de acuerdo $\square$ De acuerdo $\quad \square$ En desacuerdo $\quad \square$ Muy en desacuerdo

24. Cuando los/as profesores/as nos proponen trabajos en grupo suele haber un alto nivel de interés y participación en clase

$\square$ Muy de acuerdo $\square$ De acuerdo $\quad \square$ En desacuerdo $\quad \square$ Muy en desacuerdo

25. Los profesores/as de matemáticas están siempre dispuestos/as a prestar ayuda y a aclarar las dudas y dificultades que surjan durante la clase

$\square$ Muy de acuerdo $\quad \square$ De acuerdo $\quad \square$ En desacuerdo $\quad \square$ Muy en desacuerdo

- 70 - $\quad$ Revista Electrónica de Investigación Psicoeducativa. ISSN. 1696-2095. № 8, Vol 4 (1) 2006, pp: 47 - 72. 
26. Para mis profesores/as de matemáticas soy un/a buen/a alumno/a

$\square$ Muy de acuerdo $\quad \square$ De acuerdo $\quad \square$ En desacuerdo $\quad \square$ Muy en desacuerdo

27. Mis relaciones con los/as profesores/as de matemáticas son satisfactorias

$\square$ Muy de acuerdo $\quad \square$ De acuerdo $\quad \square$ En desacuerdo $\quad \square$ Muy en desacuerdo

28. Los/as buenos/as profesores/as que explican con bastante claridad y entusiasmo y son agradables hacen que gusten las matemáticas

$\square$ Muy de acuerdo $\quad \square$ De acuerdo $\quad \square$ En desacuerdo $\quad \square$ Muy en desacuerdo

29. Los/as profesores/as de matemáticas se interesan por mi evolución y rendimiento en la materia

$\square$ Muy de acuerdo $\quad \square$ De acuerdo $\quad \square$ En desacuerdo $\quad \square$ Muy en desacuerdo

30. En clase de matemáticas los/as profesores/as valoran mi esfuerzo y reconocen mi trabajo diario en la asignatura

$\square$ Muy de acuerdo $\quad \square$ De acuerdo $\quad \square$ En desacuerdo $\quad \square$ Muy en desacuerdo

31. Alguno de mis padres espera de mí buenos resultados en matemáticas

$\square$ Muy de acuerdo $\quad \square$ De acuerdo $\quad \square$ En desacuerdo $\quad \square$ Muy en desacuerdo

32. Las matemáticas que nos enseñan en el instituto no les interesan a mis padres

$\square$ Muy de acuerdo $\quad \square$ De acuerdo $\quad \square$ En desacuerdo $\quad \square$ Muy en desacuerdo

33. Alguno de mis padres era bastante bueno/a resolviendo problemas de matemáticas

$\square$ Muy de acuerdo $\quad \square$ De acuerdo $\quad \square$ En desacuerdo $\quad \square$ Muy en desacuerdo

34. Alguno de mis padres me anima y ayuda con los problemas de matemáticas

$\square$ Muy de acuerdo $\quad \square$ De acuerdo $\quad \square$ En desacuerdo $\quad \square$ Muy en desacuerdo

35. Mis amigos/as pasan de las matemáticas

$\square$ Muy de acuerdo $\quad \square$ De acuerdo $\quad \square$ En desacuerdo $\quad \square$ Muy en desacuerdo

36. Las matemáticas son importantes porque las profesiones más remuneradas económicamente están relacionadas con ellas

$\square$ Muy de acuerdo $\quad \square$ De acuerdo $\quad \square$ En desacuerdo $\quad \square$ Muy en desacuerdo

37. La gente a la que le gustan las matemáticas suelen ser un poco raras

$\square$ Muy de acuerdo $\quad \square$ De acuerdo $\quad \square$ En desacuerdo $\quad \square$ Muy en desacuerdo

38. El aumentar mis conocimientos matemáticos me hará sentir una persona competente en la sociedad

$\square$ Muy de acuerdo $\quad \square$ De acuerdo $\quad \square$ En desacuerdo $\quad \square$ Muy en desacuerdo

39. Las matemáticas son para cabezas inteligentes y creativas

$\square$ Muy de acuerdo $\quad \square$ De acuerdo $\quad \square$ En desacuerdo $\quad \square$ Muy en desacuerdo 
40. Dominar las matemáticas me permitirá tener éxito en mis estudios posteriores

$\square$ Muy de acuerdo $\quad \square$ De acuerdo $\quad \square$ En desacuerdo $\quad \square$ Muy en desacuerdo

41. La gente que es buena en matemáticas no tiene que gastar tiempo pensando cómo resolver un problema

$\square$ Muy de acuerdo $\quad \square$ De acuerdo $\quad \square$ En desacuerdo $\quad \square$ Muy en desacuerdo

42. Las clases de matemáticas se me hacen eternas, son muy pesadas, no estoy a gusto y siento deseos de salir corriendo

$\square$ Muy de acuerdo $\quad \square$ De acuerdo $\quad \square$ En desacuerdo $\quad \square$ Muy en desacuerdo

43. Disfruto los días que no tenemos clases de matemáticas porque no me interesan ni me atraen

$\square$ Muy de acuerdo $\quad \square$ De acuerdo $\quad \square$ En desacuerdo $\quad \square$ Muy en desacuerdo

44. Ante un problema complicado suelo darme por vencido fácilmente

$\square$ Muy de acuerdo $\quad \square$ De acuerdo $\quad \square$ En desacuerdo $\quad \square$ Muy en desacuerdo

45. Cuando me enfrento a un problema experimento mucha curiosidad por conocer la solución

$\square$ Muy de acuerdo $\quad \square$ De acuerdo $\quad \square$ En desacuerdo $\quad \square$ Muy en desacuerdo

46. Me angustio y siento miedo cuando el profesor me propone "por sorpresa" que resuelva un problema

$\square$ Muy de acuerdo $\quad \square$ De acuerdo $\quad \square$ En desacuerdo $\quad \square$ Muy en desacuerdo

47. Cuando resuelvo problemas en grupo tengo más seguridad en mí mismo/a

$\square$ Muy de acuerdo $\quad \square$ De acuerdo $\quad \square$ En desacuerdo $\quad \square$ Muy en desacuerdo

48. Cuando me atasco o bloqueo en la resolución de un problema empiezo a sentirme inseguro, desesperado, nervioso,...

$\square$ Muy de acuerdo $\quad \square$ De acuerdo $\quad \square$ En desacuerdo $\quad \square$ Muy en desacuerdo

49. Si no encuentro la solución de un problema tengo la sensación de haber fracasado y de haber perdido el tiempo

$\square$ Muy de acuerdo $\quad \square$ De acuerdo $\quad \square$ En desacuerdo $\quad \square$ Muy en desacuerdo

50. Me provoca gran satisfacción llegar a resolver con éxito un problema matemático

$\square$ Muy de acuerdo $\quad \square$ De acuerdo $\quad \square$ En desacuerdo $\quad \square$ Muy en desacuerdo

51. Cuando fracasan mis intentos por resolver un problema lo intento de nuevo

$\square$ Muy de acuerdo $\quad \square$ De acuerdo $\quad \square$ En desacuerdo $\quad \square$ Muy en desacuerdo

52. La resolución de un problema exige esfuerzo, perseverancia y paciencia

Muy de acuerdo $\quad \square$ De acuerdo $\square$ En desacuerdo $\quad \square$ Muy en desacuerdo 